\title{
Avaliação do desenvolvimento da discondroplasia tibial em frangos de corte submetidos à dieta com 25 hidroxicolecalciferol
}

\section{Evaluation of tibial dyschondroplasia development in broiler chickens fed diets containing 25 hydroxicolecalciferol}

\author{
Roselaine PONSO ${ }^{1}$; Douglas Emygdio de FARIA ${ }^{2}$; Ricardo de ALBUQUERQUE'; Ibiara Correia \\ de Lima Almeida PAZ ${ }^{1}$; Silvana Martinez Baraldi ARTONI ${ }^{4}$; Andréa Luciana dos SANTOS ${ }^{5}$; Gisele \\ SAVIANI ${ }^{3}$; Cintia Maria Monteiro de ARAÚJO ${ }^{3}$
}

\author{
${ }^{1}$ Faculdade de Ciências Agrárias da Universidade Federal da Grande Dourados, Dourados- MS, Brasil \\ ${ }^{2}$ Faculdade de Zootecnia e Engenharia de Alimentos da Universidade de São Paulo, Pirassununga-SP, Brasil \\ ${ }^{3}$ Faculdade de Medicina Veterinária e Zootecnia da Universidade de São Paulo, Pirassununga-SP, Brasil \\ ${ }^{4}$ Faculdade de Ciências Agrárias e Veterinárias da Universidade Estadual Paulista, Jaboticabal-SP, Brasil \\ ${ }^{5}$ Universidade Federal de Mato Grosso, Rondonópolis-MT, Brasil
}

\begin{abstract}
Resumo
Este trabalho teve como objetivo avaliar o desenvolvimento da discondroplasia tibial (DT) em frangos de corte de 1 a 21 dias. Foram utilizados 440 pintinhos machos de um dia, provenientes de matrizes com 60 a 62 semanas de idade, distribuídos em delineamento inteiramente casualisado em esquema fatorial $3 \times 3+2$, resultando em 11 tratamentos com quatro repetições de 10 aves cada. Os fatores estudados foram: linhagem da ave (Ross 308, Cobb 500 e Hybro), níveis e fontes de vitamina $\mathrm{D}\left(1250 \mathrm{UI} \mathrm{D}_{3} / \mathrm{kg}\right.$ sem $25-(\mathrm{OH}) \mathrm{D}_{3} ; 1250 \mathrm{UI} \mathrm{D}_{3} / \mathrm{kg}$ com $69 \mathrm{mg} 25-(\mathrm{OH}) \mathrm{D}_{3} /$ ton e $3000 \mathrm{UI} \mathrm{D} / \mathrm{kg}$ com $69 \mathrm{mg} 25-(\mathrm{OH}) \mathrm{D}_{3} /$ ton) e dois tratamentos controles com níveis de cálcio e fósforo com $3000 \mathrm{UI} \mathrm{D}_{3} / \mathrm{kg}$ sem $25-(\mathrm{OH}) \mathrm{D}_{3}$ e $3000 \mathrm{UI} \mathrm{D}_{3} / \mathrm{kg}$ com $69 \mathrm{mg} 25-(\mathrm{OH}) \mathrm{D}_{3} /$ ton de ração. Foram avaliadas características ósseas. No período de 1 a 21 dias, os resultados indicaram que as concentrações de cálcio e fósforo nas tíbias não foram influenciadas pelos tratamentos e que o consumo de ração foi superior para os tratamentos que compõem o fatorial. A resistência óssea também não foi influenciada pelos tratamentos e a análise histológica não evidenciou lesões características de DT. Conclui-se que, nas condições experimentais da presente pesquisa, o desenvolvimento de DT não foi observado.
\end{abstract}

Palavras-chave: Discondroplasia tibial. Frango de Corte. Placa de Crescimento.

\begin{abstract}
This research was carried out to evaluate the development of tibial dyschondroplasia (TD) in broilers from 1 to 21 days. Four hundred forty day-old male chickens, from broiler breeders aged 60-62 weeks, were randomly distributed in a $3 \times 3+2$ factorial arrangement, resulting in 11 treatments with four replicates of 10 birds each. The factors evaluated were: bird strains (Ross 308, Cobb 500, and Hybro), levels and sources of vitamin D (1250UI D $/ \mathrm{kg}$ without $25-(\mathrm{OH}) \mathrm{D}_{3}$; $1250 \mathrm{UI} \mathrm{D} / \mathrm{kg}$ with $69 \mathrm{mg} 25-(\mathrm{OH}) \mathrm{D}_{3} /$ ton, and $3000 \mathrm{UI} \mathrm{D}_{3} / \mathrm{kg}$ with $\left.69 \mathrm{mg} 25-(\mathrm{OH}) \mathrm{D}_{3} / \mathrm{ton}\right)$, and two control treatments containing calcium and phosphorus levels with $3000 \mathrm{UI} \mathrm{D}_{3} / \mathrm{kg}$ without $25-(\mathrm{OH}) \mathrm{D}_{3}$ and $3000 \mathrm{UI} \mathrm{D}_{3} / \mathrm{kg}$ with $69 \mathrm{mg} 25-$ $(\mathrm{OH}) \mathrm{D}_{3} /$ ton of feed. Bone characteristics were evaluated. From 1 to 21 days, the results showed that bone calcium and phosphorus concentrations were not influenced by the treatments, however, feed intake was higher for factorial than control treatments. Bone breaking resistance was not influenced by the treatments and there were no typical lesions of TD. In conclusion, it was not observed TD development in broilers from 1 to 21 days according to experimental procedures of this research.
\end{abstract}

Keywords: Tibial dyschondroplasia. Broiler chickens. Growth plate.

\section{Introdução}

$\mathrm{Na}$ avicultura industrial existe uma constante busca de conhecimento das diferentes afecções e dos procedimentos técnicos para preveni-las ou curá-las, permitindo viabilizar a intensificação da produção animal. Em outras áreas do conhecimento, como o

\section{Correspondência para:}

Roselaine Ponso

Rua Bela Vista, 190, Jardim São Pedro.

Dourados, Mato Grosso do Sul, MS, Brasil

CEP: 79810-030

e-mail: roseponsovet@hotmail.com

Cel: (67) 8192-5384

Recebido: 05/11/2010

Aprovado: 18/04/2012 
melhoramento genético, a determinação das necessidades nutricionais e o balanceamento das rações também foram fundamentais e tem permitido um aumento da eficiência zootécnica desses animais ${ }^{1}$. Além da fundamental contribuição do melhoramento genético, o sucesso da avicultura de corte decorre, em grande parte, da nutrição. Nas últimas décadas, o ganho de peso médio diário aumentou de 20 para mais de $50 \mathrm{~g} /$ dia e a idade de abate reduziu de 12 para seis semanas ${ }^{2}$. No entanto, o desenvolvimento do tecido ósseo não tem acompanhado estes processos fisiológicos, aumentando a incidência de problemas locomotores e fragilidade óssea. A compreensão dos inúmeros fatores nutricionais envolvidos no desenvolvimento ósseo, como deficiências de proteínas e aminoácidos, vitaminas e minerais, são de suma importância na ocorrência de desordens esqueléticas.

Com a seleção genética voltada para o alto ganho de peso e elevadas taxas de crescimento, tem-se depositado grandes cargas sobre ossos e juntas relativamente imaturos, causando assim má formação óssea e, consequentemente, problemas de pernas, dada a falta de exercícios. Além disso, a atividade locomotora é drasticamente reduzida no final do período de criação em frangos selecionados para um rápido crescimento³.

A Discondroplasia Tibial (DT) é uma anormalidade metabólica de frangos de corte em crescimento causada pela formação de uma massa anormal de cartilagem não vascularizada, pouco mineralizada na metáfise da extremidade proximal da tíbia. Essa cartilagem é uma persistência da cartilagem pré-hipertrófica que não foi invadida pelos vasos da metáfise e que não sofreu calcificação, ou uma assincronia na diferenciação dos condrócitos, ocasionando uma camada de condrócitos pré-hipertróficos e de uma cartilagem na tíbia proximal não calcificada e resistente à invasão vascular, que se estende distalmente na placa de crescimento da metáfise proximal da tíbia e ocasionalmente, na metáfise distal e proximal do tarsometatarso e proximal do fêmur e úmero ${ }^{4,5}$.
O uso da vitamina $\mathrm{D}_{3}$ na avicultura tem despertado grande interesse dos pesquisadores nos últimos anos devido, principalmente, à sua capacidade de induzir a diferenciação de condrócitos, controle do crescimento ósseo, prevenção do raquitismo e diminuição da incidência de $\mathrm{DT}^{6}$. Recentes pesquisas têm apontado exigências de vitamina $D_{3}$ na ordem de $6,9 \mu \mathrm{g} / \mathrm{kg}$ para crescimento, $10,1 \mu \mathrm{g} / \mathrm{kg}$ para aumentar o conteúdo de cinzas dos ossos, $13,8 \mu \mathrm{g} / \mathrm{kg}$ para aumentar a quantidade de cálcio no sangue, e $22,6 \mu \mathrm{g} / \mathrm{kg}$ para prevenir o raquitismo ${ }^{7}$. Thorp et al. ${ }^{8}$ mostraram marcante redução da DT em frangos alimentados com diferentes níveis de 1,25-dihidroxicolicalciferol, $1,25(\mathrm{OH})_{2} \mathrm{D}_{3}$. Os níveis utilizados nesse trabalho foram baseados nessa literatura citada e segundo Rostagno, Albino e Donzele ${ }^{10}$ tendo também sido o usual nas granjas. Os estudos de microscopia eletrônica de varredura indicam hipertrofia incompleta do condrócitos, onde foi possível verificar que os condrócitos atingem apenas $40 \%$ do tamanho esperado, entretanto, em estudos realizados com microscopia eletrônica de transmissão, ocorrem mudanças degenerativas que incluem dilatação do retículo endoplasmático e das cisternas de Golgi, inchaço mitocondrial e núcleo picnótico?.

O objetivo deste trabalho foi avaliar o desempenho e o desenvolvimento da DT em frangos de corte de diferentes linhagens, alimentados com dietas contendo diferentes fontes e níveis de vitamina $\mathrm{D}$, até os 21 dias de idade. Dessa forma, propõe-se avaliar se as mudanças ultraestruturais na placa de crescimento de frango de corte com predisposição para DT podem ser identificadas antes dos 21 dias, possibilitando o controle precoce desta patologia que vem ocasionando perdas significativas na produção de frangos de corte.

\section{Material e Método}

O experimento foi conduzido no aviário experimental da Faculdade de Medicina Veterinária e Zootecnia da Universidade de São Paulo, localiza- 
do no Campus de Pirassununga - SP, com duração de 21 dias. Foram utilizados 440 pintinhos de 1 dia, machos, das linhagens Ross 308, Cobb 500 e Hybro, cujos pesos médios com 1 dia de idade foram de 47 g, $46 \mathrm{~g}$, e de $48 \mathrm{~g}$ respectivamente, provenientes de matrizes com 60 a 62 semanas de idade, criados em baterias metálicas. O programa de luz foi de 24 horas de luz/dia. As temperaturas (máxima e mínima) no interior do aviário foram registradas diariamente, com a utilização de um termômetro analógico e os dados foram obtidos por leituras no início da manhã e no final da tarde.

As aves foram distribuídas em 11 tratamentos, obedecendo a um delineamento inteiramente casualisado em esquema fatorial $3 \times 3+2$, ou seja, três linhagens, três níveis e fontes de vitamina $\mathrm{D}$ e dois tratamentos controle, resultando em 11 tratamentos com quatro repetições de 10 aves por tratamento.

Os fatores analisados foram: linhagem da ave (Ross 308, Cobb 500 e Hybro), níveis e fontes de vitamina $\mathrm{D}\left(1250 \mathrm{UI} \mathrm{D}_{3} / \mathrm{kg} \mathrm{sem} 25-(\mathrm{OH}) \mathrm{D}_{3} ; 1250 \mathrm{UI} \mathrm{D}_{3} / \mathrm{kg}\right.$ com $69 \mathrm{mg} 25-(\mathrm{OH}) \mathrm{D}_{3} /$ ton e $3000 \mathrm{UI} \mathrm{D}_{3} / \mathrm{kg}$ com $69 \mathrm{mg} 25-$ $(\mathrm{OH}) \mathrm{D}_{3} /$ ton) e dois tratamentos controles com níveis de cálcio e fósforo segundo Rostagno, Albino e Donzele ${ }^{10} \mathrm{com} 3000 \mathrm{UI} \mathrm{D}_{3} / \mathrm{kg}$ sem $25-(\mathrm{OH}) \mathrm{D}_{3}$ e $3000 \mathrm{UI}$ $\mathrm{D}_{3} / \mathrm{kg}$ com 69mg 25- $(\mathrm{OH}) \mathrm{D}_{3} /$ ton de ração.

Para a preparação do galpão, foram instalados sistema de aquecimento dos pintinhos, além da montagem das baterias e posterior distribuição aleatória das parcelas experimentais. As aves foram alimentadas diariamente com dietas formuladas à base de milho e farelo de soja, de acordo com as exigências para as duas fases de criação, pré-inicial e inicial, segundo as recomendações de Rostagno, Albino e Donzele ${ }^{10}$ exceto para $\mathrm{Ca}$ e $\mathrm{P}$. As composições nutricionais calculadas das dietas experimentais nas fases pré-inicial e inicial, são indicadas nas tabelas 1 e 2 , respectivamente. A linhagem Ross foi escolhida como tratamento controle por ter sido a mesma utilizada por Flemming, Jansen e Endo ${ }^{11}$.
Para as análises histológicas, foram colhidas amostras da tíbia de uma ave por repetição de tratamento aos 7, 14, e 21 dias de idade. As aves foram sacrificadas por deslocamento cervical, quando se procedeu a retirada do material para resistência, análises histológicas e microscopia eletrônica de varredura. As tíbias foram removidas e dissecadas. A tíbia direita foi imediatamente fixada para histologia e microscopia eletrônica e a tíbia esquerda congelada para análise de resistência à quebra e de cinzas. As tíbias direitas foram incisadas com bisturi em cortes longitudinais, divididas em paquímeros ventral e dorsal, sendo o paquímero ventral imerso em fixador primário de glutarealdeído 2,5\% e o paquímero dorsal imerso em solução fixadora de Bouin para estudos de morfologia. A solução fixadora de Bouin foi preparada para que atingisse uma concentração de $2,5 \%$ de formol. Para cada $100 \mathrm{ml}$ dessa solução, utilizou-se $75 \mathrm{~mL}$ de ácido pícrico, $25 \mathrm{~mL}$ de formol a $40 \%$ e $5 \mathrm{ml}$ de ácido acético glacial. Os fragmentos ósseos coletados para análise permaneceram nessa solução por 24 horas. Em seguida, foram mantidos em álcool $70 \%$ até o momento do processamento. O material fixado em solução fixadora de Bouin seguiu a rotina de inclusão em parafina e corte em micrótomo Spencer de secções de $5 \mu \mathrm{m}$, onde foram corados por hematoxilina-eosina para a observação e descrição histológica dos ossos. As lâminas foram analisadas em microscopia óptica, obtendo-se imagens por meio do programa Kontron KS 4003.0 em microscópio Olympus DX 60 acoplado a câmara Axio CAN HRc, utilizando o programa de análise de imagem (KONTRON KS 400 Zeiss).

No paquímero dorsal das tíbias foram realizadas análises histológicas. As amostras foram analisadas e não foram achados dados significativos, portanto, para confecção das imagens de microscopia eletrônica foram colhidas aleatoriamente, e da região caudo-medial da placa de crescimento de cada tíbia, foi excisado, cortado em fragmentos e imersos em fixador primário de cacodilato por três horas. As 
Tabela 1 - Composição e níveis nutricionais calculados das dietas experimentais. Pré-Inicial (1 a 7 dias)

\begin{tabular}{|c|c|c|c|c|c|}
\hline \multicolumn{6}{|c|}{ Tratamentos } \\
\hline Ingredientes & T1, T4 e T7 & T2, T5 e T8 & T3, T6 e T9 & T10 & T11 \\
\hline Milho & 61,003 & 60,901 & 60,799 & 60,275 & 60,173 \\
\hline Farelo de soja (45\%) & 34,566 & 34,584 & 34,603 & 34,697 & 34,715 \\
\hline Calcário & 0,429 & 0,429 & 0,429 & 0,897 & 0,896 \\
\hline Fosfato bicálcico & 2,028 & 2,028 & 2,028 & 1,867 & 1,868 \\
\hline Sal & 0,497 & 0,497 & 0,497 & 0,497 & 0,497 \\
\hline Óleo vegetal & 0,291 & 0,325 & 0,359 & 0,533 & 0,566 \\
\hline L-Lisina HCL & 0,427 & 0,426 & 0,426 & 0,424 & 0,424 \\
\hline DL-Metionina & 0,373 & 0,373 & 0,373 & 0,374 & 0,374 \\
\hline L-Treonina & 0,187 & 0,187 & 0,186 & 0,186 & 0,186 \\
\hline Supl.Vitamínico/mineral ${ }^{1}$ & 0,200 & 0,200 & 0,200 & 0,200 & 0,200 \\
\hline 25 Hidroxi (Hy D) ${ }^{2}$ & - & 0,050 & 0,050 & - & 0,050 \\
\hline Colecalciferol $\left(\mathrm{D}_{3}\right)^{3}$ & _- & _- & 0,050 & 0,050 & 0,050 \\
\hline Total & 100,000 & 100,000 & 100,000 & 100,000 & 100,000 \\
\hline \multicolumn{6}{|c|}{ Níveis nutricionais (\%) } \\
\hline E.M. $(\mathrm{kcal} / \mathrm{kg})$ & 2.960 & 2.960 & 2.960 & 2.960 & 2.960 \\
\hline Proteína bruta (\%) & 22,11 & 22,11 & 22,11 & 22,11 & 22,11 \\
\hline Cálcio (\%) & 0,80 & 0,80 & 0,80 & 0.94 & 0,94 \\
\hline Fósforo disponível & 0,50 & 0,50 & 0,50 & 0,47 & 0,47 \\
\hline Sódio & 0,22 & 0,22 & 0,22 & 0,22 & 0,22 \\
\hline Met+cis digestível & 0,96 & 0,96 & 0,96 & 0,96 & 0,96 \\
\hline Metionina digestível & 0,66 & 0,66 & 0,66 & 0,66 & 0,66 \\
\hline Lisina digestível & 1,36 & 1,36 & 1,36 & 1,36 & 1,36 \\
\hline Treonina digestível & 0,88 & 0,88 & 0,88 & 0,88 & 0,88 \\
\hline Triptofano digestível & 0,23 & 0,23 & 0,23 & 0,23 & 0,23 \\
\hline
\end{tabular}

${ }^{1}$ Suplemento Vitamínico - mineral + aditivos $=(2 \mathrm{~kg} / \mathrm{ton})$ proporcionando $1250 \mathrm{UI} \mathrm{D} / \mathrm{Kg}$ de ração. Quantidade por kg de ração: Vit. A9000 UI; Vit. D - 1250 UI; Vit. E- 20 mg; Vit. K - 2,5 mg; Vit. B - 1,5 mg; Vit. B - 6 mg; Vit. B - 3 mg; Vit. B - 12 mcg; Niacina- 25 mg; Àcido Pantotênico- 12 mg; Biotina- 60 mcg; Acido Fólico- 0,8 mg; Selênio- 0,25 mg; Ferro- 50 mg; Cobre- 10 mg; Zinco- 50 mg; Manganês- 80 mg; Iodo- $1 \mathrm{mg}$; Cobalto- 1mg; Antioxidante (BHT)- 120 mg; Veículo q.s.p. 1000 g. ${ }^{2}$ Pré-mistura de Hy.D (0,5 kg/ton) proporcionando 69 mg de $25(\mathrm{OH})\left(\mathrm{D}_{3}\right) /$ ton ração. ${ }^{3}$ Pré-mistura de colecalciferol $\left(\mathrm{D}_{3}\right)(0.5 \mathrm{~kg} /$ ton $)$ proporcionando $1750 \mathrm{UI} \mathrm{D}_{3} / \mathrm{kg}$ de ração

peças foram enxaguadas e pós-fixadas em tetróxido de ósmio a $2 \%$ diluído em cacodilato, e contrastadas em acetato de uranila, desidratadas em séries crescentes de álcool, embebidas em óxido de propileno incluídas em araldite. Cortes semi-finos foram feitos usando um ultramicrotomo e corados com azul de toluidina, observados ao microscópio de transmissão no Instituto de Ciências Biomédicas da Universidade de São Paulo.

Para a microscopia eletrônica, os fragmentos ósseos foram coletados e acondicionados em frascos contendo solução conservadora de glutaraldeído $4 \%$. Depois foram lavados por seis vezes consecutivas em tampão fosfato $0,1 \mathrm{M}, \mathrm{pH} 7,2$ por três horas e então pós-fixados em solução de tetróxido de ósmio a $2 \%$ durante 30 minutos, à temperatura de $4^{\circ} \mathrm{C}$. Posteriormente, as amostras foram novamente lavadas com o mesmo tampão por seis vezes consecutivas, desidratadas em concentrações crescentes de álcool etílico (30, 50, 70, 80, 90 e 100\%), durante 20 minutos em cada concentração, sendo que, na última, as amostras foram lavadas por três vezes consecutivas, 20 minutos em cada lavagem. Depois de cada desidratação, o material passou pela câmara de secagem do secador de ponto crítico, mediante a utilização de dióxido de carbono. O material foi então montado em porta objeto apropriado, recoberto com uma camada de $30 \mathrm{~nm}$ de ouro e, finalmente, elétromicrografado em microscópio eletrônico de varredura (Modelo Jeol JSM 54 10), operando em $15 \mathrm{KV}$. 
Tabela 2 - Composição e níveis nutricionais calculados das dietas experimentais. Inicial (8 - 21 Dias)

\begin{tabular}{|c|c|c|c|c|c|}
\hline \multicolumn{6}{|c|}{ Tratamentos } \\
\hline Ingredientes & T1, T4 e T7 & T2, T5 e T8 & T3, T6 e T9 & T10 & T11 \\
\hline Milho & 61,701 & 61,600 & 61,498 & 61,264 & 61,162 \\
\hline Farelo de soja (45\%) & 33,016 & 33,034 & 33,052 & 33,094 & 33,113 \\
\hline Calcário & 0,432 & 0,432 & 0,431 & 0,866 & 0,866 \\
\hline Fosfato bicálcico & 2,043 & 2,043 & 2,044 & 1,774 & 1,774 \\
\hline Sal & 0,497 & 0,497 & 0,497 & 0,497 & 0,497 \\
\hline Óleo vegetal & 1,495 & 1,529 & 1,563 & 1,640 & 1,674 \\
\hline L-Lisina HCL & 0,258 & 0,258 & 0,257 & 0,257 & 0,256 \\
\hline DL-Metionina & 0,267 & 0,267 & 0,267 & 0,267 & 0,267 \\
\hline L-Treonina & 0.091 & 0,091 & 0,091 & 0,091 & 0,091 \\
\hline Supl.Vitamínico/minerall & 0,200 & 0,200 & 0,200 & 0,200 & 0,200 \\
\hline 25 Hidroxi (Hy D) 2 & - & 0,050 & 0,050 & - & 0,050 \\
\hline Colecalciferol (D3)3 & - & - & 0,050 & 0,050 & 0,050 \\
\hline Total & 100,000 & 100,000 & 100,000 & 100,000 & 100,000 \\
\hline \multicolumn{6}{|c|}{ Níveis nutricionais (\%) } \\
\hline E.M. (kcal/kg) & 3.050 & 3.050 & 3.050 & 3.050 & 3.050 \\
\hline Proteína bruta & 21,14 & 21,14 & 21,14 & 21,14 & 21,14 \\
\hline Cálcio & 0,80 & 0,80 & 0,80 & 0,90 & 0,90 \\
\hline Fósforo disponível & 0,50 & 0,50 & 0,50 & 0,45 & 0,45 \\
\hline Sódio & 0,22 & 0,22 & 0,22 & 0,22 & 0,22 \\
\hline Met+cis digestível & 0,84 & 0,84 & 0,84 & 0,84 & 0,84 \\
\hline Metionina digestível & 0,55 & 0,55 & 0,55 & 0,55 & 0,55 \\
\hline Lisina digestível & 1,19 & 1,19 & 1,19 & 1,19 & 1,19 \\
\hline Treonina digestível & 0,77 & 0,77 & 0,77 & 0,77 & 0,77 \\
\hline Triptofano digestível & 0,22 & 0,22 & 0,22 & 0,22 & 0,22 \\
\hline
\end{tabular}

${ }^{1}$ Suplemento Vitamínico - mineral + aditivos $=(2 \mathrm{~kg} / \mathrm{ton})$ proporcionando $1250 \mathrm{UI} \mathrm{D}_{\mathrm{V}} / \mathrm{Kg}$ de ração. Quantidade por kg de ração: Vit. A9000 UI; Vit. D - 1250 UI; Vit. E- 20 mg; Vit. K - 2,5 mg; Vit. B - 1,5 mg; Vit. B - 6 mg; Vit. B - 3 mg; Vit. B - 12 mcg; Niacina- 25 mg; Àcido Pantotênico- 12 mg; Biotina- $60 \mathrm{mcg}$; Acido Fólico- 0,8 mg; Selênio- 0,25 mg; Ferro- $50 \mathrm{mg}$; Cobre- $10 \mathrm{mg}$; Zinco- 50 mg; Manganês- 80 mg; Iodo- $1 \mathrm{mg}$; Cobalto- $1 \mathrm{mg}$; Antioxidante (BHT)- $120 \mathrm{mg}$; Veículo q.s.p. 1000 g. ${ }^{2}$ Pré-mistura de Hy.D (0,5 kg/ton) proporcionando 69 mg de $25(\mathrm{OH})\left(\mathrm{D}_{3}\right) /$ ton ração. ${ }^{3}$ Pré-mistura de colecalciferol $\left(\mathrm{D}_{3}\right)(0.5 \mathrm{~kg} / \mathrm{ton})$ proporcionando $1750 \mathrm{UI} \mathrm{D}_{3} / \mathrm{kg}$ de ração

A determinação das cinzas foi realizada no Laboratório de Bromatologia do Departamento de Zootecnia da Faculdade de Zootecnia e Engenharia de Alimentos (FZEA/USP). Os ossos foram secos em estufa a $105^{\circ} \mathrm{C}$, por 12 horas, e desengordurados em aparelho extrator "soxchlet" por 5 horas. Posteriormente, foram colocados na estufa ventilada a $60^{\circ} \mathrm{C}$ por 12 horas para posterior pesagem dos ossos secos e desengordurados. Em seguida, foram moídos em moinho de bola, retiradas amostras e colocadas em mufla a $600^{\circ} \mathrm{C}$, durante 4 horas, para determinação dos teores de cinzas, cálcio e fósforo, de acordo com a metodologia proposta por Silva ${ }^{12}$.

O desdobramento dos graus de liberdade do modelo para estudar os efeitos principais e de interação dos níveis dos fatores foi feito através de contrastes ortogonais (Tabela 3), conforme orientações sugeridas pela Fapesp, após o último relatório apresentado. Na presença de interação, foi realizado um estudo de regressão das variáveis respostas em função dos níveis de vitamina $\mathrm{D}$, para cada uma das linhagens. Nas análises estatísticas, utilizou-se o procedimento GLM do programa Statistical Analisys System ${ }^{13}$.

\section{Resultados e Discussão}

Nas figuras 1A e 1B, observa-se a formação da cartilagem, com penetração vascular, não caracterizando a presença de discondroplasia tibial, assim como a zona de transição e início de formação do 
Tabela 3 - Contrastes ortogonais utilizados na comparação dos dados das diversas características

\begin{tabular}{|c|c|c|c|c|c|c|c|c|c|c|c|}
\hline \multirow{2}{*}{$\begin{array}{c}\text { Linhagem } \\
\text { Vitamina } D^{3}\end{array}$} & \multicolumn{3}{|c|}{ Ross } & \multicolumn{3}{|c|}{ Cobb } & \multicolumn{3}{|c|}{ Hybro } & \multirow{2}{*}{$\begin{array}{l}\text { Ross } \\
3000\end{array}$} & \multirow{2}{*}{$\begin{array}{l}\text { Ross } \\
3000\end{array}$} \\
\hline & 1250 & 1250 & 3000 & 1250 & 1250 & 3000 & 1250 & 1250 & 3000 & & \\
\hline HyD & Sem & com & com & Sem & com & com & sem & com & com & Sem & com \\
\hline Tratamento & T1 & $\mathrm{T} 2$ & T3 & T4 & T5 & T6 & T7 & T8 & T9 & T10 & T11 \\
\hline C1: (Controles vs. Fatorial) & -2 & -2 & -2 & -2 & -2 & -2 & -2 & -2 & -2 & 9 & 9 \\
\hline C2: (Controle 1 vs. Controle 2) & 0 & 0 & 0 & 0 & 0 & 0 & 0 & 0 & 0 & 1 & -1 \\
\hline \multirow{2}{*}{ C3: (Linhagem) } & -2 & -2 & -2 & 1 & 1 & 1 & 1 & 1 & 1 & 0 & 0 \\
\hline & 0 & 0 & 0 & 1 & 1 & 1 & -1 & -1 & -1 & 0 & 0 \\
\hline \multirow{2}{*}{ C4: (Vitamina D) } & 2 & -1 & -1 & 2 & -1 & -1 & 2 & -1 & -1 & 0 & 0 \\
\hline & 0 & 1 & -1 & 0 & 1 & -1 & 0 & 1 & -1 & 0 & 0 \\
\hline \multirow{4}{*}{ C5: (Interação LxV) } & -4 & 2 & 2 & 2 & -1 & -1 & 2 & -1 & -1 & 0 & 0 \\
\hline & 0 & -2 & 2 & 0 & 1 & -1 & 0 & 1 & -1 & 0 & 0 \\
\hline & 0 & 0 & 0 & 2 & -1 & -1 & -2 & 1 & 1 & 0 & 0 \\
\hline & 0 & 0 & 0 & 0 & 1 & -1 & 0 & -1 & 1 & 0 & 0 \\
\hline
\end{tabular}

tecido osteoide, mineralização e zona de transição entre cartilagem e osso. Essa caracterização de tecido ósseo normal sem a presença de lesões concernentes a DT está de acordo com as afirmações de Thorp et al. ${ }^{8}$ que mostraram marcante redução da DT em frangos alimentados com diferentes níveis de 1,25-dihidroxicolicalciferol $1,25(\mathrm{OH})_{2} \mathrm{D}_{3}$, revelando que a placa de crescimento da tíbia mostrou acúmulos de condrócitos transicionais, o que confirma a hipótese de que a causa da discondroplasia seja uma falha na diferenciação de condrócitos. Farquharson et al. ${ }^{14}$ relata que $1,25(\mathrm{OH})_{2} \mathrm{D}_{3}$ previne a DT e que ela pode aumentar a taxa de diferenciação de condrócitos nas placas de crescimento, estando de acordo com os relatos de Kevin e Edwards ${ }^{15}$, que acrescentam que a forma ativa da vitamina $\mathrm{D}$, a $1,25-(\mathrm{OH})_{2} \mathrm{D}$ é um importante regulador do desenvolvimento do tecido ósseo. Ledwaba e Roberson ${ }^{16}$ relataram a eficiência dos metabólitos 1-hidroxilados na promoção de uma adequada diferenciação de condrócitos, afirmando que a suplementação com a 1,25-dihidroxivitamina D3 $1,25(\mathrm{OH})_{2} \mathrm{D}_{3}$ evita completamente a discondroplasia tibial, entretanto, esse produto não se encontra disponível comercialmente no Brasil.
Nas figuras 1C e 1D, ao exame de microscopia eletrônica de varredura, observou-se a extremidade proximal de tíbias de frangos de corte aos 21 dias de idade, submetidos à dieta com 25 hidroxicolecalciferol, nas concentrações de $3000 \mathrm{UI} / \mathrm{kg}$. Nota-se o tecido ósseo normal, e estrutura trabecular no tecido denso. As fibras paralelas, com intensa concentração de tecido cartilaginoso, identificando a integridade da linha de crescimento, e presença constante de vasos sanguíneos, confirmando ausência de DT, o que corrobora o descrito por Gonzales e Macari ${ }^{5}$ de que a lesão da DT é avascular, podendo ser notadas algumas áreas de necrose. Os resultados indicam presença de vasos sanguíneos, tecido ósseo e cartilaginoso, hipertrofia completa do condrócitos, que atingiram o tamanho esperado, e não sofreram mudanças degenerativas, confirmando os resultados de Bains, Brake e Pardue ${ }^{9}$, entretanto, estes relatam dilatação do retículo endoplasmático e das cisternas de Golgi, edema mitocondrial e núcleo picnótico, o que não foi observado nessa pesquisa, por ter sido utilizado microscopia eletrônica de varredura, sendo possível analisar apenas estruturas de superfície e não organelas, como foi relatado pelos autores, que utilizaram microscópio de transmissão. 

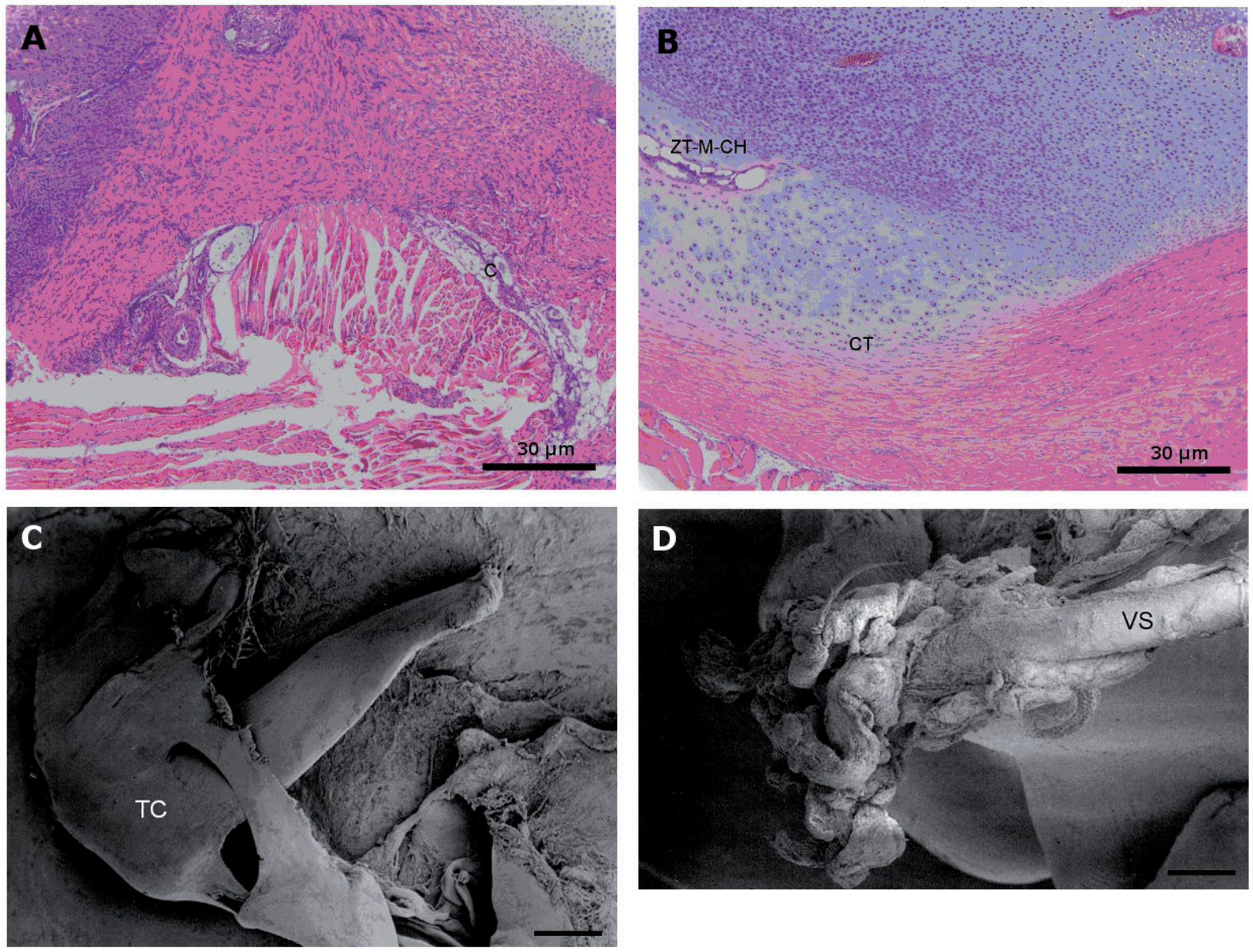

Figura 1 - A) T2R2 (Tratamento 2 - Repetição 2) - 14 dias de idade. Fotomicrografia de corte transversal da tíbia, descalcificada e corada pela técnica da hematoxilina e eosina. Observa-se a formação da cartilagem da fise, com penetração vascular (C), não caracterizando a presença de discondroplasia tibial. Aumento de 100x. B) T5R4 (Tratamento 5 - Repetição 4) - 14 dias de idade. Fotomicrografia de corte transversal da tíbia, descalcificada e corada pela técnica da hematoxilina e eosina. Observa-se a zona de transição (ZT) e início de formação do tecido osteoide e de mineralização, canal de Havers (TO-M-CH). Aumento de 100x. C) T3R4 - Eletromicrografia de Varredura, de osso de frango aos 21 dias, ilustrando tecido ósseo com a presença de tecido cartilaginoso (TC) em sua extremidade. 1.500x. D) T6R3 - Eletromicrografia de Varredura, de osso de frango aos 21 dias, ilustrando a presença de vaso sanguíneo (VS), demonstrando vascularização normal, penetrando na zona de crescimento. 1500x

Os valores médios e erros padrões do período de 1 a 21 dias das concentrações de cálcio e fósforo estão indicados nas tabelas 4 e 5 , respectivamente. Para análise dos níveis de cálcio e fósforo, foram utilizados os mesmos níveis apontados por Edwards ${ }^{17}$ para cálcio, que observa que níveis adequados de cálcio $(0,80 \%)$ e baixos de fósforo total ( $0,50 \%)$, com exposição de luz ultravioleta, diminuem a incidência de DT e quando as dietas contêm níveis adequados de cálcio, indicam que a síntese de colecalciferol é mais ativa quando é administrado na ração, e de Ledwaba e Roberson ${ }^{16}$, que observaram que valores de $0,65 \%$ e $0,50 \%$ de cálcio e fósforo total, respectivamente, associados com alguns níveis de suplementação de $25(\mathrm{OH})_{2} \mathrm{D}_{3}$, ou menos de $69 \mu \mathrm{g}$ deste elemento são efetivos para prevenir a discondroplasia tibial.

Aos sete dias, a linhagem Ross apresentou maior concentração de cálcio, seguida pela linhagem Cobb e Hybro. Já aos 14 dias, a linhagem Hybro apresentou a maior concentração de cálcio, juntamente com a linhagem Cobb, e aos 21 dias, com $1250 \mathrm{UI} \mathrm{D}_{3} / \mathrm{kg}$, a maior deposição de cálcio foi verificada na linhagem Hybro e 
Tabela 4 - Valores médios e erros padrões (e.p.) das concentrações de cálcio por mg/kg na matéria original (tíbia) de 1 a 21 dias

\begin{tabular}{|c|c|c|c|c|c|c|c|c|c|c|c|c|}
\hline Linhagem & & Ross & & & Cobb & & & Hybro & & Ross & Ross & \\
\hline HyD & 1250 & 1250 & 3000 & 1250 & 1250 & 3000 & 1250 & 1250 & 3000 & 3000 & 3000 & e.p. \\
\hline Vitamina D & sem & com & com & sem & com & com & sem & com & Com & sem & com & \\
\hline Cálcio 7 dias & 7,02 & 7,62 & 8,49 & 6,55 & 7,31 & 6,19 & 6,79 & 7,23 & 5,83 & 5,96 & 6,08 & 0,54 \\
\hline Cálcio 14 dias & 4,46 & 5,29 & 4,70 & 4,76 & 4,61 & 5,13 & 5,33 & 5,58 & 5,82 & 5,23 & 4,97 & 0,31 \\
\hline Cálcio 21 dias & 10,06 & 10,84 & 9,44 & 9,52 & 10,93 & 12,02 & 11,37 & 11,87 & 11,96 & 12,23 & 11,65 & 0,87 \\
\hline
\end{tabular}

Tabela 5 - Valores médios e erros padrões (e.p.) das concentrações de fósforo por mg/kg na matéria original (tíbia) de 1 a 21 dias

\begin{tabular}{|c|c|c|c|c|c|c|c|c|c|c|c|c|}
\hline Linhagem & & Ross & & & Cobb & & & Hybro & & Ross & Ross & \multirow{3}{*}{ e.p. } \\
\hline HyD & 1250 & 1250 & 3000 & 1250 & 1250 & 3000 & 1250 & 1250 & 3000 & 3000 & 3000 & \\
\hline Vitamina D & Sem & Com & com & sem & com & com & sem & com & com & sem & Com & \\
\hline Fósforo 7 dias & 4,40 & 4,77 & 5,57 & 4,37 & 4,59 & 4,08 & 5,33 & 4,71 & 4,03 & 4,12 & 4,32 & 0,26 \\
\hline Fósforo 14 dias & 2,58 & 2,78 & 2,61 & 2,72 & 2,61 & 3,30 & 3,07 & 3,44 & 3,51 & 3,15 & 3,53 & 0,22 \\
\hline Fósforo 21 dias & 5,72 & 5,55 & 5,30 & 5,57 & 5,44 & 6,29 & 6,07 & 5,71 & 6,89 & 5,80 & 6,11 & 0,43 \\
\hline
\end{tabular}

com $3000 \mathrm{UI} \mathrm{D}_{3} / \mathrm{kg}$, as linhagens Cobb e Hybro tiveram maiores deposições com valores próximos, $12,02 \%$ e 11,96\%, respectivamente, sendo os efeitos não significativos. Aos sete dias, a linhagem Ross apresentou maior concentração de fósforo, seguida pela linhagem Hybro e Cobb, diferentemente das outras linhagens que tiveram resultados não significativos com relação a concentrações de fósforo quando suplementadas com o maior nível do produto. Já aos 14 dias, a linhagem Hybro apresentou a maior concentração de fósforo, juntamente com a linhagem Cobb. Aos 21 dias, com 1250 UI $\mathrm{D}_{3} / \mathrm{kg}$, a maior concentração de fósforo foi verificada na linhagem Hybro e com $3000 \mathrm{UI} \mathrm{D}_{3} / \mathrm{kg}$, as linhagens Hybro e Cobb tiveram maiores concentrações com valores próximos, 6,89\% e 6,29\%, respectivamente, apresentando resultado não significativo, embora tenham tido uma concentração linear de deposição de fósforo.

De acordo com $\mathrm{NRC}^{18}$, as exigências de cálcio para frangos são de $1 \%$ de 1 a 21 dias e $0,90 \%$ de 22 a 42 dias. No presente trabalho, nos tratamentos adicionais (controle) foram utilizados os níveis de cálcio e fósforo, segundo recomendações de Rostagno, Albino e Donzele ${ }^{10}$ ou seja, 0,94 e 0,90\% de cálcio para pré-inicial e inicial, e 0,47 e $0,45 \%$ de fósforo disponível para pré-inicial e inicial, respectivamente. Leske e Coon ${ }^{19}$ são unânimes em afirmar a necessidade da adequada suplementação desses minerais durante a fase de crescimento, pois, do contrário acarretará em desequilíbrio na homeostase mineral e insuficiente desenvolvimento dos ossos, sendo essa uma das origens da calcificação inadequada. Underwood e Suttle ${ }^{20}$ relatam que a deposição mais intensa de cálcio ocorre na fase de crescimento, podendo chegar ao final de um mês a $80 \%$ do cálcio total numa ave adulta. A utilização de cálcio, fósforo e vitamina $\mathrm{D}$ também foram objeto de pesquisa de Sá, Gomes e Albino. ${ }^{21}$, que relataram estar esses elementos diretamente relacionados ao metabolismo animal, entretanto, no presente trabalho, em todos os tratamentos, as tíbias apresentaram características histológicas normais.

Considerando a dinâmica do melhoramento genético das aves referidos nos diversos trabalhos sobre o tema em questão, futuros estudos são indicados com a mesma abordagem metodológica, mas simulando as condições de campo com as aves sendo criadas em piso, visando compatibilizar a pesquisa com as condições de produção comercial de frangos de corte. 


\section{Conclusões}

Com base nos resultados obtidos, é licito concluir que a utilização de 1250 ou 3000 UI do metabólito 25 hidroxicolecalciferol/kg de ração não influencia as características ósseas (morfologia e concentração de Ca e P), de frangos das linhagens Cobb, Ross e Hybro, nem interfere no desenvolvimento de lesões compatíveis com a discondroplasia tibial, no período de 1 a 21 dias de idade. Essa ausência de alterações inerentes

\section{Referências}

1. ALBUQUERQUE, R. Antimicrobianos como promotores de crescimento. In: PALERMO, J.; SPINOSA, H. S.; GÓRNIAK, S. L. (Ed.). Farmacologia aplicada à avicultura. São Paulo: Roca, 2005. cap. 9, p. 149-159.

2. SILVA JÚNIOR, R. G. C.; LANA, G. R. Q.; RABELLO, C. B.; BARBOSA W. A.; LANA, S. R. V. Exigência de metionina + cistina para frangos de corte machos de 1 a 21 e de 22 a 42 dias de idade, em clima tropical. Revista Brasileira Zootecnia, v. 34 , p. 2399-2407, 2005. (supl.).

3. ALMEIDA, P. I. C .L.; MENDES, A. A.; ALMEIDA, L.; NAAS, I. A.; MENEZES, A. G.; MARTINS, M. R. F. B.; FERNANDES, B. C. S.; MILBRADT, E. L.; BALOG, A.; KOMIYAMA, C. M.; CARDOSO, K. F. G. Avaliação da degeneração femoral e bem-estar em frangos de corte. Revista Brasileira de Ciência Avícola, supl. 10, p. 1, 2008.

4. ALMEIDA, P. I. C. L.; MENDES, A. A.; TAKITA, T. S.; VULCANO, L. C.; GUERRA, P. C.; WESCHELER, F. S.; GARCIA, R. G. Tibial dyschondroplasia and bone mineral density. Brazilian Journal of Poultry Science, v. 6, n. 4, p. 207-212, 2004.

5. GONZALES, E.; MACARI, M. Problemas locomotores em frangos de corte. In: SIMPÓSIO BRASIL SUL DE AVICUlTURA, 7., 2006, Chapecó-SC. Anais... Chapecó, 2006. p. 79-92.

6. WHITEHEAD, C. C.; CORMACK, H. A.; TEIR, M. C. L; .FLEMING, R. H. High vitamin D requirements $\mathrm{n}$ broilers for bone quality and prevention of tibial dyschondroplasia and interactions with dietary calcium, available phosphorus and vitam A. British Poultry Science, v. 3, p. 425 -436, 2004.

7. BAKER, D. H.; BIEHL, R. R.; EMMERT, J. L. Vitamin $\mathrm{D}_{3}$ requirement of young chicks receiving diets varying in calcium, and available phosphorus. British Poultry Science, v. 39, p. 413-417, 1998.

8. THORP, B. H.; DUCRO, B.; WHITEHEAD, C. C.; FARQUHARSON, C.; SORENSEN, P. Avian tibial dyschondroplasia: the interaction of genetic selection and dietary 1,25 dihydroxycolecalciferol. Avian Pathology, v. 22, p. 311-324, 1993 .

9. BAINS, B. S.; BRAKE, J. T; PARDUE, S. L. Reducing leg weakness in commercial broilers. World Poultry, v. 14, n. 1, p. 24-27, 1998. ao quadro de DT possivelmente se dá pelo fato desse distúrbio aparecer frequentemente entre a $3^{\circ}$ e $8^{\circ}$ semana de idade em aves criadas em piso, e os resultados apresentados referem-se a aves de até 21 dias, criadas em baterias metálicas.

\section{Agradecimentos}

Fundação de Amparo à Pesquisa - Fapesp, DSM Nutritional Products.

10. ROSTAGNO, H. S.; ALBINO, L. F. T.; DONZELE, J. Tabelas brasileiras para aves e suinos: composição de alimentos e exigências nutricionais. Viçosa: UFV, 2005. 141 p.

11.FLEMMING, J. S.; JANZEN, S. A.; ENDO, M. A. Rendimento de carcaças em linhagens de frangos de corte. Archives of Veterinary Science, v. 41, p. 67-63, 1999.

12.SILVA, D.J. Análises de alimentos: métodos químicos e biológicos. 2. ed. Viçosa: UFV, 1998. 165 p.

13.STATISTICAL ANALISYS SYSTEM. SAS user's guide: statistics. Version 8. Cary: SAS, 2001.

14.FARQUHARSON, C.; WHITEHEAD, C. C.; RENNIE, J. S. LOVERIDGE, N. In vivo effects of 1,25 dihidroxycolecalfiferol on the proliferation and differentiation of avian chondrocytees. Journal of Bone and Mineral Research, n. 8, p. 1081-1088, 1983.

15.KEVIN, D. R.; EDWARDS, H. M. Effect of dietary 1,25-dihydroxycholecalciferol level on broiler performance. Poultry Science, v. 75, p. 90-94, 1996.

16.LEDWABA, F. M.; ROBERSON, K. D. Effectiveness of 25-hidroxycholecalciferol in the prevention of tibial dyschondroplasia in Ross cockerels depends on dietary calcium level. Poultry Science, v. 82, p. 1769-1777, 2003.

17.EDWARDS, H. M. Nutrition and skeletal problems in poultry. Poultry Science, v. 79, p. 1018-1023, 2000.

18. NATIONAL RESEARCH COUNCIL - NRC. Nutrient requirements of poultry. 9. ed. Washington: National Academy Science, 1994. 155 p.

19.LESKE, J. L.; COON, C.N. A biossay to determine the effect of phitase phosphorus retention off feed ingredients as determined with broilers and laying hens. Poultry Science, v. 78, n. 8, p. 1151-1157, 1999.

20.UNDERWOOD, E.J.; SUTTLE, N.F. Mineral nutrition of livestock. 3. ed. London: CAB International, 1999. 614 p.

21.SÁ, L. M.; GOMES, P. C.; ALBINO, L. F. T. Exigências nutricionais de cálcio e sua biodisponibilidade em alguns alimentos para frangos de corte no período de 1 a 21 dias de idade. Revista da Sociedade Brasileira de Zootecnia, v. 33, n. 1, p. 157-168, 2004. 